\title{
PAPER \\ On-Demand Generalization of Road Networks Based on Facility Search Results
}

\author{
Daisuke YAMAMOTO $^{\dagger \text { a) }}$,Member, Masaki MURASE ${ }^{\dagger}$, Nonmember, and Naohisa TAKAHASHI ${ }^{\dagger}$, Member $^{2}$
}

\begin{abstract}
SUMMARY Road generalization is a method for thinning out road networks to allow easy viewing according to the size of the map. Most conventional road generalization methods mainly focus on the length of a stroke, which is a chain of links with good continuity based on the principle of perceptual grouping applied to network data such as roads and rivers. However, in the case of facility search in a web map service, for example, a "restaurant guide map," a road generalization mechanism can be more effective if it depends not only on the stroke length but also on the facility search results. Accordingly, in this study, we implement an on-demand road generalization method that adapts to both the facility search results and the stroke length. Moreover, a sufficiently fast response speed is achieved for practical use in web map services. In particular, this study proposes a fat-stroke model that links facility information to individual strokes and implements a road generalization method that uses this model to improve the response time. In addition, we develop a prototype based on the proposed system. The system evaluation results are based on three indicators, namely, response time of the road generalization system, connectivity between strokes, and connectivity between stroke and facilities. Our experimental results suggest that the proposed method can yield improved response times by a factor of 100 or more while affording higher connectivity.

key words: road generalization, web map service, stroke
\end{abstract}

\section{Introduction}

Web map services such as Google Maps and OpenStreetMap*have witnessed widespread use in recent years. These maps deliver greater visual utility by "sorting out" roads to be drawn according to the scale of the map. In general, road generalization refers to drawing thinned-out road networks to improve visual utility. In this context, Thomson and Richardson proposed the concept of the stroke [1], which is a chain of links with good continuity based on the principle of perceptual grouping applied to network data such as roads and rivers, and they developed a road generalization method based on the stroke length. Subsequently, many types of road generalization methods such as connection analysis and information-theory-based road generalization have been proposed [1], [2].

In web map services, facility search results can be superimposed on the map. For example, when a user searches for the category "restaurants" on a map, facilities with restaurants are displayed on the map with corresponding markers such as pins. However, roads leading to facilities

\footnotetext{
Manuscript received December 15, 2017.

Manuscript revised September 3, 2018.

Manuscript publicized October 16, 2018.

${ }^{\dagger}$ The authors are with the Nagoya Institute of Technology, Nagoya-shi, 466-8555 Japan.

a) E-mail: yamamoto.daisuke@ @itech.ac.jp

DOI: 10.1587/transinf.2017EDP7405
}

have not been considered in conventional road generalization methods despite the fact that the roads to the facility are important for facility access. This can be particularly problematic in the case of printing map search results (say, restaurant maps) for distribution.

Accordingly, researchers have suggested that implementing an on-demand road generalization method that not only considers the stroke length but also adapts to the facility search results will likely solve the abovementioned problem. Therefore, in our proposed method, a road generalization mechanism is implemented by prioritizing roads connecting to facilities. Moreover, in existing road generalization methods, it is common to perform offline processing in advance; therefore, the response speed required for ondemand processing has not been considered.

Against this backdrop, we aim to implement an ondemand road generalization method that considers stroke length and adapts to search results, thereby ensuring that the processing speed is sufficiently fast to be of practical use for web map services. The main problems in implementing the proposed system are summarized below.

Problem 1 Although the aim of the proposed method is to implement road generalization that adapts to both stroke length and facility search results, most conventional road generalization methods take only stroke length into account.

Problem 2 In conventional stroke generation, the road network is analyzed independently of the facility location. To consider facility search results, it is necessary to analyze the positional relationship between the stroke and the facility after generating the stroke. Because this process is very time-consuming, the problem of slow response speed arises.

Problem 3 The visual utility of the map decreases unless a suitable number of roads are displayed according to the map size. Accordingly, it is necessary to determine the number of roads to be drawn for road generalization.

To solve the aforementioned problems, we propose the fat-stroke model, which links facility information to the respective strokes and implements a road generalization system based on the proposed model. Further, the model affords improved response time [4]. The academically innovative aspects of this research are described below:

1. Our approach enables dynamic road generalization ac-

*http://openstreetmap.org/ 


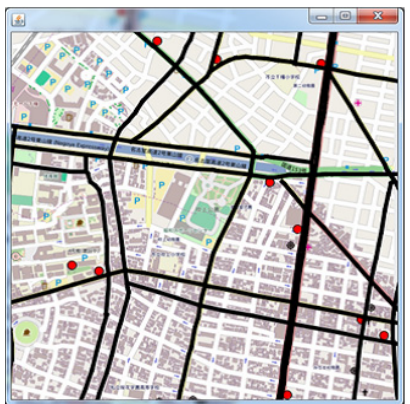

(a) Convenience store

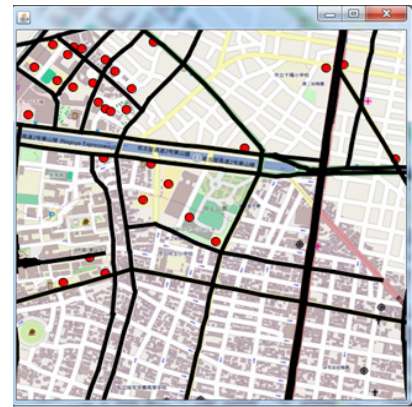

(b) Parking lot
Fig. 1 Road generalization results for different categories selected.

cording to the facility search results. In the past, road generalization has been realized using only the stroke length, but the proposed method realizes road generalization based on the stroke length and the number of facilities adjacent to the stroke.

2. Via calculating the fat-stroke in advance and storing it in the database, our method can acquire fat-strokes at high speeds. As a result, the response speed of the proposed system is greatly improved.

3. The proposed method can adjust the amount of roads to be rendered according to several parameters to be described later.

In terms of practical industrial application of the proposed system, our approach can contribute to the automatic generation of illustration maps according to search results of facility categories, such as the restaurant map that was conventionally created manually. Specifically, we assume that the illustration map is used as a web map service that can be dynamically generated.

An example of road generalization implemented on the basis of the proposed system is shown below. Figure 1 illustrates how the roads are displayed such that they are connected to the facilities in the search results as far as possible without changing the total length of the roads displayed when a search is conducted for a specific category.

These implementations not only provide a more manageable web map service but may also contribute to the field of geographic information systems (through applications in map services such as community information infrastructure) or research on cognitive maps.

\section{Related Work}

As regards implementing road generalization, Thomson and Richardson proposed the concept of stroke [1], [2] with regard to road networks. A stroke is a chain of road links that continue along a road, and it is identified according to the principle of perceptual grouping. The relationship between the road network and the stroke network is shown in Fig. 2. The road network consists of connected links between a node representing an intersection and other adjacent nodes. Figure 2 (a) shows a road network and Fig. 2 (b)

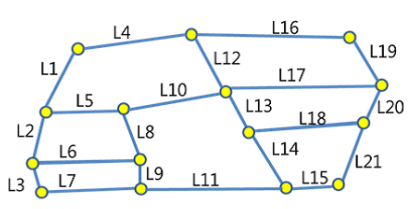

(a) Road network

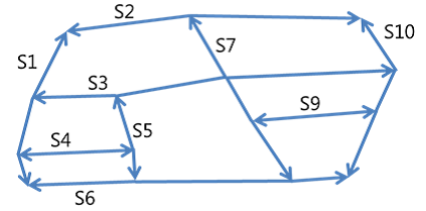

(b) Stroke network
Fig. 2 Road and stroke networks

shows the stroke network consisting of strokes generated by applying perceptual grouping to the road network. Strokes can be represented by a chain of road links. For example, in Fig. 2 (b), stroke S3 comprises links L5, L10, and L17 shown in Fig. 2 (a), and it is expressed as S3 = (L5, L10, L17).

The basic idea of implementing road generalization based on strokes originates from the empirical observation that the longer is the stroke, the more important is the road. This means that it is a mechanism through which roads are selected on the basis of priority determined by stroke length.

In this context, Zhang proposed a characteristic road selection method based on connection analysis [3]. In this method, a characteristic road is selected on the basis of the number of roads connected to it at an intersection. Subsequently, several similar methods have been proposed with regard to road generalization, wherein the relative importance of the road is estimated and the order of the road is determined on the basis of a combination of other road attributes and the length and connectivity relationship. Bjrke proposed an algorithm [8] for deselecting roads on a map on the basis of information theory. In this method, a similarity function is calculated from an arbitrary point in the road network, which is called the information point. The similarity function is calculated on the basis of the distance between two arbitrary points. Subsequently, the relationship between the similarity function and the conditional probability is derived, which facilitates optimum road generalization using mutual information. Hu et al. [9] proposed a method for selective deletion of roads using the proportion of a block (size) at a certain point with respect to the length of the road surrounding it, which they called "mesh density." The blocks with high mesh density are omitted, because the road intervals become narrower as the mesh density increases. This facilitates the creation of a small-scale road network. Jinang and Claramunt proposed a generalization model for characteristic road selection [10] in urban road networks. This model uses a structural representation of the road network, called a "connectivity graph." Furthermore, programming contests on constrained map generalization [11] have been held at ACM SIGSPATIAL in recent years. Other road generalization methods include the focus+glue+context-type fisheye view map [12], where only a part of the map, i.e., glue, is used.

Several previous research studies have focused on the generation of guide maps [5]-[7]. A guide map system performs not only road generalization but also deforming and reallocating roads, and allocating the textural labels. 
With regard to further developments in this context, Ninomiya et al. [13] proposed a method to draw a guide route on a deformed map from the perspective of a cognitive map. Kono et al. [14] proposed a method to render deformed maps for easy viewing on mobile terminals. In addition, Matsuo et al. [15] proposed a method of extracting geographical features related to deformation map retrieval. While these approaches attempt to simplify and draw a route for directions, our method extracts multiple important roads from the entire map, which is a primary difference between our approach and previous ones. Meanwhile, some previous studies have focused on map generalization for not only drawing roads but also drawing buildings. Wei et al. [16] proposed a collaborative displacement method combining aggregation, elimination, and constrained reshaping in order to solve all possible spatial conflicts during urban building map generalization. Zhao et al. [17] proposed practical techniques for solving overlapping arrangements of buildings and roads. Lee et al. [18] proposed a method of classifying buildings using machine learning for the purpose of the map generalization.

\section{Overview of Proposed System}

\subsection{Data Used}

Figure 3 shows the road data structure definition utilized in this study. When a road is treated as a network connecting intersections via points and arcs, the physical shape of the road can be represented using nodes and links. A point refers to an actual coordinate point on the road, whereas an arc refers to a series of points representing the road shape. Nodes represent the intersections of the road network, and links represent the connections between nodes. Table 1 summarizes the structure of the road table for storing these road data sets. The road data of OpenStreetMap and facility data were used in this study.

Table 2 summarizes the structure of the facility data comprising the latitude/longitude of the facility and its category. The facility category refers to the type of facility, such as restaurants and parking lots.

In addition, a loop road is created from the road data. As shown in Fig. 4, the smallest area surrounded by links is

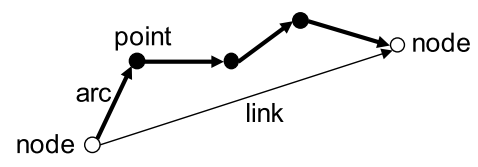

Fig. 3 Structure of road data

Table 1 Road table scheme

\begin{tabular}{l|l|l}
\multicolumn{1}{c|}{ Column } & \multicolumn{1}{|c}{ Data type } & \multicolumn{1}{c}{ Description } \\
\hline id & int & Link ID uniquely identifying a link \\
source & int & Node ID at start of the link \\
target & int & Node ID at end of the link \\
km & double & Arc length (km) \\
geom_way & geometry(lineString) & Represents link shape (arc) \\
\hline
\end{tabular}

called a block, and the links that surround a section constitute a loop road. Table 4 summarizes the data structure of a loop road table. In our study, the algorithm proposed by Yamamoto et al. [19] was used for generating loop roads.

\subsection{Configuration of Proposed System}

Figure 5 depicts the proposed system consisting of fat-stroke generation and a road generalization server. In fat-stroke generation, the loop roads and strokes are first generated from road data, and a fat stroke is generated by combining these data with the facility data. The generated information is stored in a database. These processes can be executed

Table 2 Facility table

\begin{tabular}{l|l|l}
\hline Column & \multicolumn{1}{|c|}{ Data type } & \multicolumn{1}{c}{ Description } \\
\hline id & int & $\begin{array}{l}\text { Facility ID uniquely identifying a } \\
\text { facility }\end{array}$ \\
$\begin{array}{l}\text { category } \\
\text { location }\end{array}$ & $\begin{array}{l}\text { text } \\
\text { geometry(point) }\end{array}$ & $\begin{array}{l}\text { Fatity category } \\
\text { Latide and longitude at facility lo- } \\
\text { cation }\end{array}$ \\
\hline
\end{tabular}

Table 3 Loop road table

\begin{tabular}{l|l|l}
\hline Column & Data type & \multicolumn{1}{c}{ Description } \\
\hline id & int & $\begin{array}{l}\text { Loop road ID for unique identifica- } \\
\text { tion } \\
\text { Polygon representing loop road }\end{array}$ \\
\hline
\end{tabular}

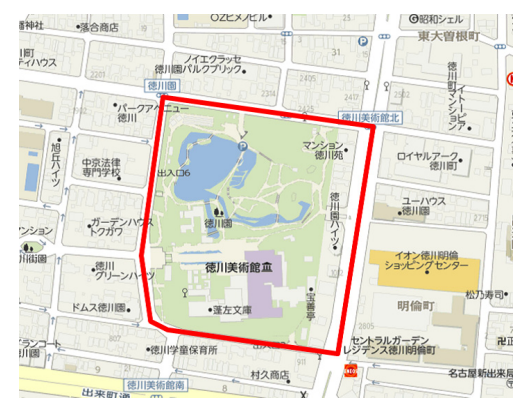

Fig. 4 Loop road around Tokugawaen

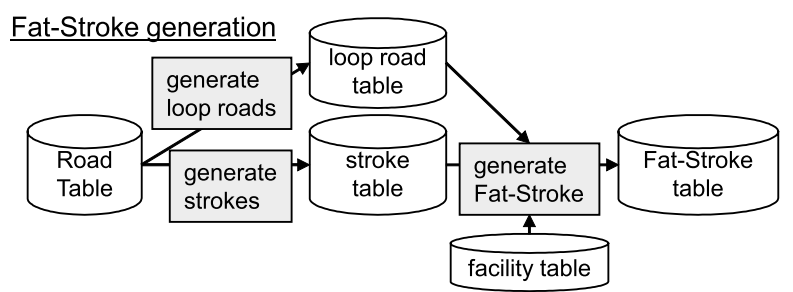

Road generalization server

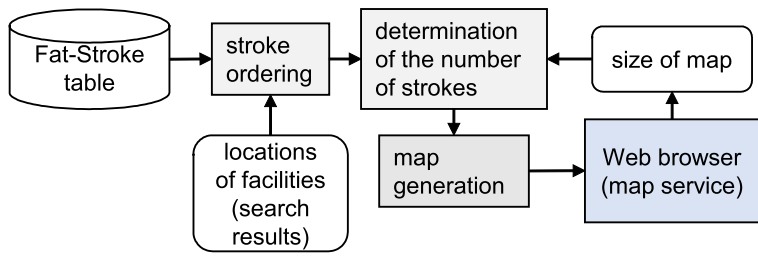

Fig. 5 Configuration of proposed system 
offline in advance.

The advantages of associating facility information with strokes rather than road links are described below. In general, the condition for good road generalization is that while important roads need to be drawn, individual roads also need to be suitably connected. Because a stroke is longer than the road, there is a high possibility that a road (stroke) will be connected rather than using a road link.

In the road generalization server, on-demand road generalization is performed in accordance with a user request. Specifically, strokes are prioritized according to the facility search results, and the number of strokes is determined according to the size of the map. Next, the stroke data are sent to the client.

Here, we note that we did not adopt the tiling method to implement road generalization in our study; because the tiling method involves displaying a map by arranging static map images in a tile pattern, it is not possible to selectively draw individual strokes. The result of the road generalization should instead be drawn by a vector map. In Figs. 1, $10,11,12$, and 16 , the results of the road generalization are drawn with black vector lines on the tiling map in order to easily evaluate the relationship with the existing map.

The PostgreSQL database and PostGIS ${ }^{\dagger}$ were used in our study; PostGIS is an extension function for handling geospatial information in PostgreSQL.

\section{Proposed Method}

This section describes the implementation of the proposed system. We describe the fat-stroke generation method proposed in this study after describing the stroke generation method. Subsequently, we describe the proposed fat-strokebased road generalization method.

\subsection{Stroke Generation}

A stroke is implemented on the basis of road links. In the proposed system, a stroke is generated on the basis of the concept of "good continuity" under the perceptual grouping principle. Table 4 summarizes the stroke data structure. First, the following symbols and functions are defined for an arbitrary link L in the road table. Next, as shown in Fig. 7, link $\mathrm{L}$ is connected to $\mathrm{k}$ links at the end point.

- $L S=L_{1}, L_{2}, \ldots, L_{i}, \ldots, L_{k}$ : set of links connected to $L$.

- $L S_{i}=L_{i 1}, L_{i 2}, \ldots, L_{i j}, \ldots, L_{i k_{i}}:$ set of links connected to $L_{i}$

- angle(A,B): outputs the angle between links A and B

- minAngle(A,S): outputs the minimum value of angle(A, B) for an arbitrary link B in link set S

Next, the two following parameters are defined:

- $\Delta$ : allowable error.

- $\delta$ : upper limit of $\theta_{i}$ for good continuation judgment (set

\footnotetext{
${ }^{\dagger}$ http://postgis.net/
}

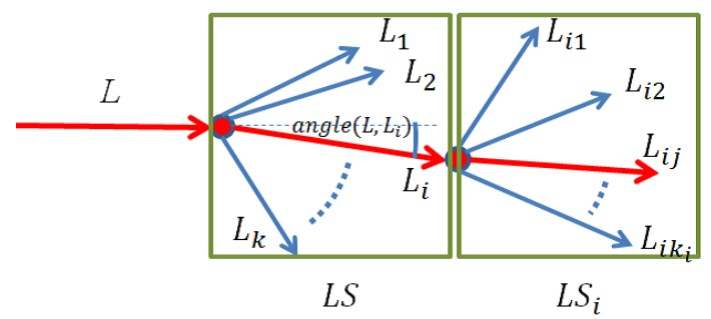

Fig. 6 Following road links corresponding to link L

\begin{tabular}{|c|c|c|}
\hline column & type & description \\
\hline $\overline{\text { stroke_id }}$ & $\overline{\text { int }}$ & Stroke ID \\
\hline $\begin{array}{l}\text { stroke_line } \\
\text { stroke_length }\end{array}$ & $\begin{array}{l}\text { MultilineString } \\
\text { double }\end{array}$ & $\begin{array}{l}\text { Represents stroke shape } \\
\text { Stroke length }\end{array}$ \\
\hline
\end{tabular}

(a)
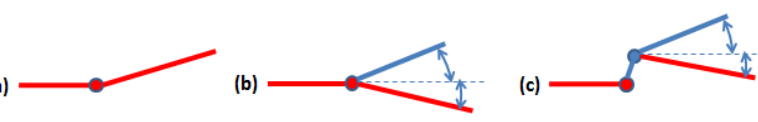

Fig. 7 Conditions for grouping links in a stroke

\section{to 45 degree)}

The rules for recognizing consecutive links as strokes are defined below. Links $L, L_{i}$, and $L_{i j}$ connected in series as shown in Fig. 6 are grouped under the same stroke based on conditions (a)-(c) depicted in Fig. 7.

(a) $L S=\left\{L_{1}\right\}$

$\rightarrow L$ and $L_{1}$ belong to the same stroke

(b) $L S=\left\{L_{1}, \ldots, L_{k}\right\}(k \geq 2)$

and $\operatorname{angle}\left(L, L_{i}\right)<\delta$

and $\operatorname{minAngle}(L, L S)=L_{i}$

$\rightarrow L$ and $L_{i}$ belong to the same stroke

(c) $L S=\left\{L_{1}, \ldots, L_{k}\right\}(k \geq 2)$

and angle $\left(L, L_{i j}\right)<\delta$

and minAngle $(L, L S)=L_{i j}$

$\rightarrow L$ and $L_{i j}$ belong to the same stroke

\subsection{Fat-Stroke Generation}

A fat stroke is generated from a stroke, loop road, and facility data by means of the procedure shown in Fig. 8. There are three intermediate tables required for generating a fat stroke. The first one is the LF table, which relates the loop road and facility data. The LF table stores the set of road data and the data of the facilities along the loop road. The second one is the SL table, which relates the stroke and the loop road. The SL table stores the data on the stroke and the loop road in its vicinity. The third one is the SF table, which stores data on the stroke and the facilities linked with the stroke. The fat-stroke generation procedure is as follows:

(i) For each loop road $L R_{i}\left(i=1, \ldots, N_{L R}\right.$, where $N_{L R}$ denotes the number of loop roads in the loop road table) in the loop road table, obtain all of the facility data inside $L R_{i}$ and place them into the loop road and facility 


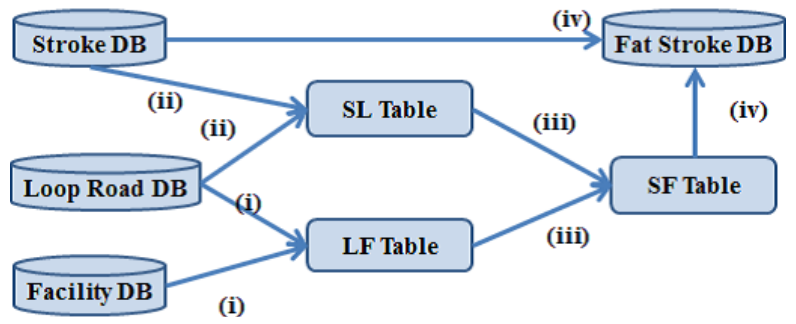

Fig. 8 Fat-stroke generation procedure

\begin{tabular}{lll}
\multicolumn{2}{c}{ Table 5 } & LF table \\
\hline column & type & description \\
\hline looproad_id & int & Loop road ID \\
facility_id & int & Facility ID \\
facility_category & int & Facility category \\
\hline
\end{tabular}

\begin{tabular}{lll}
\multicolumn{2}{c}{ Table 6 } & SL table \\
\hline column & type & description \\
\hline stroke_id & int & Stroke ID \\
looproad_id & int & Loop road ID \\
\hline
\end{tabular}

\begin{tabular}{lll}
\multicolumn{2}{c}{ Table 7 } & SF table \\
\hline column & type & description \\
\hline stroke_id & int & Stroke ID \\
facility_id & int & Facility ID \\
facility_category & int & Faclity category \\
\hline
\end{tabular}

table, as listed in Table 5 (Fig. 8 (i)). Execute the following SQL query :

select looproad_id, facility_id from

looproad_table, facility_table where

st_contains(LR_i, facility_location)\};

(ii) For each stroke $S_{j}\left(j=1, \ldots, N_{s s}\right.$, where $N_{s s}$ represents the number of strokes in the stroke table) in the stroke table, find the loop road including the links of stroke $S_{j}$. As a result, the SL table indicating the relationship between the stroke and the loop road is created as in Table 6 (Fig. 8(ii)). Execute the following SQL query:

select stroke_id, looproad_id from stroke_table, looproad_table where st_intersects (S_j, looproad_poly);

(iii) Create the table (SF table) that stores the relationship between the stroke and the facilities connected to it (Table 7) from the relationship tables created in (i) and (ii) (Fig. 8 (iii))

(iv) Finally, the fat-stroke DB (Table 8) is generated from the stroke DB and the SF table (Fig. 8 (iv)).

The stroke shown in red in Fig. 9 is associated with six loop roads, and seven facilities are linked with the strokes. Therefore, for the stroke shown in Fig. 9, facility_num $=7$.

\begin{tabular}{lll} 
& Table 8 & Fat-Stroke data structure \\
\hline column & type & description \\
\hline stroke_id & int & Stroke ID \\
stroke_length & double & Stroke length \\
facility_num & int & Number of facilities linked to this stroke \\
facility_category & int & Facility category \\
\hline
\end{tabular}

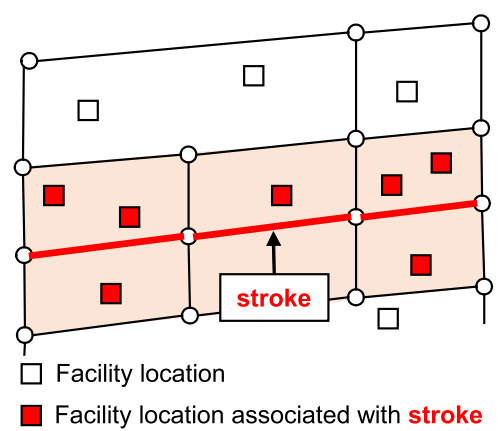

Fig. 9 Fat stroke example

\subsection{Road Generalization Method}

\subsubsection{Stroke Ordering}

In general, selecting important roads (strokes) from the following viewpoint has been considered to be suitable for road generalization.

Long following road A road that continues over a long distance is often a major road such as a national highway or a prefectural road. Major roads are important because the passage of people or vehicular traffic on these roads is usually high, and they are most likely to be on routes to many target facilities.

Many facilities in target category along the road A road with many target facilities, or with many facilities related to the target category, is important because it is on a route to many target facilities.

Roads passing through important points Roads passing through important points, such as the current location, should be selected.

Accordingly, the importance of the stroke is determined by considering the stroke length and the number of facilities linked with the stroke. The following symbols are defined for the stroke set $s \in S$.

- $s_{f}$ : number of facilities in search results, linked to stroke $s$

- $s_{l e n}$ : length of stroke $s$

- $w_{f}$ : weighting for the facility

- $w_{s}$ : weighting for the stroke $\left(w_{f}+w_{s}=1\right)$

- Near $(l)$ : the stroke nearest to location $l$

- $M S L$ : the important places such as the current spot designated by the user (optional)

The importance $S_{s}$ of stroke $s$ is expressed using the above symbols. 


$$
S_{s}=\left\{\begin{array}{l}
1 \quad \text { if } s=N e a r(l) \text { where } l \in M S L) \\
\frac{s_{f}}{\sum_{s \in S} s_{f}} w_{f}+\frac{s_{\text {len }}}{\sum_{s \in S} s_{l e n}} w_{s} \quad \text { (otherwise) }
\end{array}\right.
$$

The importance of the stroke can be adjusted by changing the ratio of $w_{f}, w_{s}$, which determines whether priority is given to the stroke or the number of facilities; $w_{f}, w_{s}$ are system parameters.

In the above calculations, we do not consider importance or accessibility of each facility in order to simplify the problem. On the other hand, it is also important to consider the importance of the facility. In this case, $s_{f}$ can be calculated in consideration of the weight $f_{w}$ of facility $f$ as follows:

$$
s_{f}=\sum_{f \in \text { facilities linked to stroke s }} f_{w}
$$

\subsubsection{Determining the Number of Strokes}

The number of strokes to be drawn according to the size of the map is determined by the proportion of the strokes drawn (road coverage rate). With higher road coverage rates, the intervals between the strokes become narrower, thereby degrading the visual usefulness of the map. Conversely, if the coverage is too small, the amount of information on the map is greatly reduced, making it difficult to comprehend. The actual road coverage rate $\alpha(n)$ for the stroke drawn on the map is obtained from the following equation with respect to the reference value $\alpha_{0}, a(n) \leq a_{0}$, where $n$ denotes the number of strokes drawn.

$$
\alpha(n)=\frac{\sum_{i=0}^{n} s_{i_{l e n}} s_{w i d t h}}{A} \leq \alpha_{o}
$$

where,

- $s_{i}$ : ith stroke in order of importance

- $s_{i_{l e n}}$ : length of stroke $s_{i}$

- $s_{\text {width }}$ : width of stroke $s$ (system parameter)

- $A$ : area under map display range

- $\alpha_{0}$ : reference road coverage rate(system parameter)

Here, $\alpha_{0}$ indicates the rate of the area of the road in the entire map. The area of the road can be obtained as the product of the length of the road and the width of the line used for drawing the road. Parameter $s_{\text {width }}$ indicates the thickness of the road line drawn on the map. When $\alpha_{0}$ is constant, $s_{\text {width }}$ has the following effects. Increasing the value of $s_{\text {width }}$ decreases the number of strokes drawn as the lines of the road become thicker. In this case, decreasing $s_{\text {width }}$ increases the number of strokes. For example, in the case of an illustration map, the road should be drawn "thick" in order to display the road in an easy-to-understand manner. Consequently, $s_{\text {width }}$ should be set to a high value.

\section{Implementation}

\subsection{Road Generalization System Implementation}

The results of road generalization over a range of latitudes (a) $700 p x \times 700 p x$

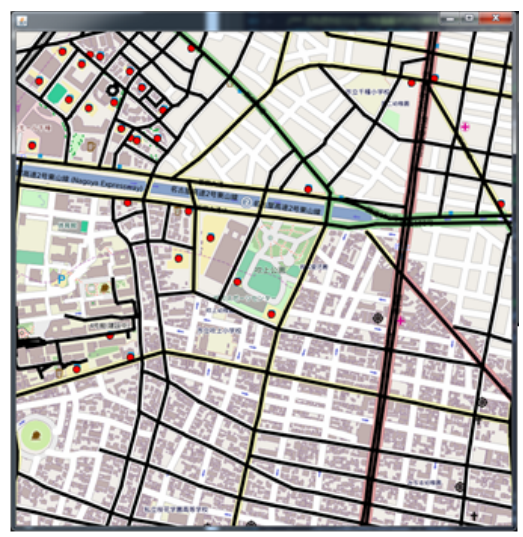

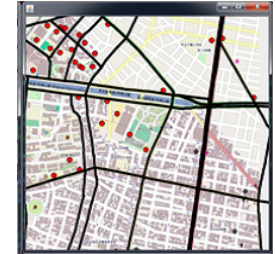

(b) $350 p x \times 350 p x$
Fig. 10 Results of map generalization for two different map sizes

(136.924-136.943) and longitudes (35.146-35.162498) are shown in Fig. 10. When the user reduces the screen size of the map, Fig. 10 (a) changes to Fig. 10 (b). The red dots indicate the facility locations, and the black lines represent the strokes. In this case, the system parameters were set as follows.

$$
w_{f}=0.5, w_{s}=0.5, s_{\text {width }}=5, \alpha_{0}=0.15
$$

The size of the map in Fig. 10 (a) is $A=700 \times 700 p x$, and the number of strokes selected is 67 . The size of the map in Fig. 10 (b) is $A=350 \times 350 p x$, and the number of strokes selected is 17. Although both these maps represent the same range of area with the same target category selected, the map sizes differ. The number of strokes drawn is reduced so as to not degrade the visual usefulness as the screen size of the map is reduced.

The results of three road generalizations with different $\alpha_{0}$ values are shown in Fig. 11. The number of strokes drawn on the map changes according to the value of $\alpha_{0}$. Figure 1 shows the results of road generalization for different categories of facilities selected. Figure 1 (a) shows the results of selecting "convenience store" as a facility category, whereas Fig. 1 (b) shows the result when "parking lot" is selected. These figures demonstrate that the selected strokes change as the selected facility category changes.

\subsection{Road Generalization System Implementation Using Web Map}

We next developed a web map system that performs ondemand road generalization using the fat stroke. The Leaflet ${ }^{\dagger}$ JavaScript framework was used for implementing the web map service. In Fig. 12, the map on the right shows the facilities in a category and the related strokes of high importance obtained by selecting a category from the menu on the left. The result of road generalization varies according to the results for the category selected. Moreover, the display result of the stroke changes with the size of the map.

\footnotetext{
${ }^{\dagger}$ http://leafletjs.com/
} 


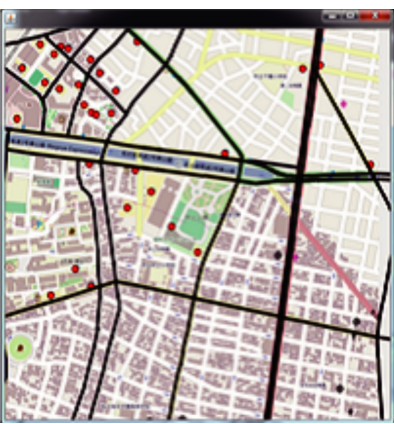

(a) $\alpha_{0}=0.075$

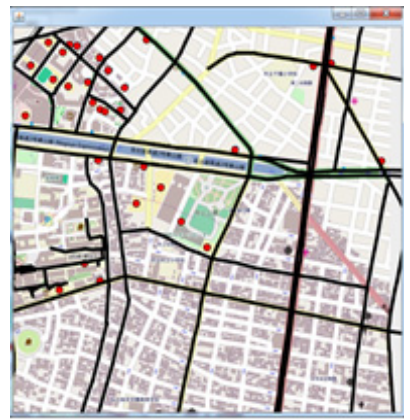

(b) $\alpha_{0}=0.15$

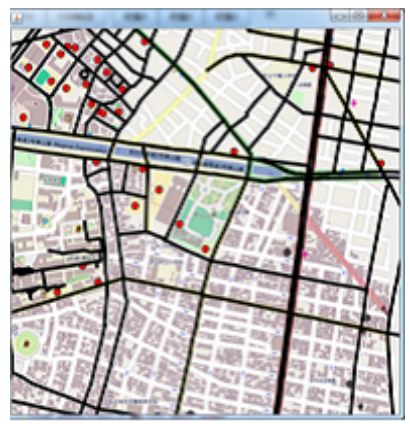

(c) $\alpha_{0}=0.2$

Fig. 11 Generalization results for different $\alpha_{0}$ values

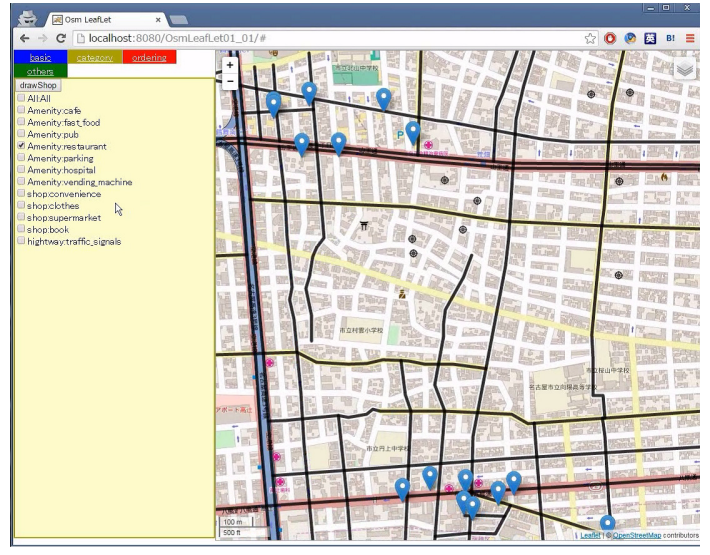

Fig. 12 Road generalization system for web map

\section{Evaluation}

\subsection{Evaluation of Response Time}

Existing web map services such as Google Maps use a tiling method, where map images are generated in advance, thereby allowing a fast response time. In contrast, in the proposed system, because it is necessary to generate a map every time a request from the road database is made, in general, a longer processing time is required compared to the aforementioned method. Therefore, in our study, we measured the response time for the proposed system to assess whether a map can be generated sufficiently fast to ensure an acceptable response time for a web map system. In the proposed method, a model that stores the pre-computed fat stroke in the database is employed. Our aim here is to demonstrate the merits of such an approach.

\subsubsection{Evaluation Method}

The response speed is evaluated by changing the size of the map for a target location at (latitude, longitude $)=(35.154$, 136.933). The two following methods are compared and assessed here. The scale is 1:25000.

- Method 1: Road generalization without using fat- stroke DB

- Method 2: Road generalization using fat-stroke DB (proposed).

The time from data acquisition to generalization completion for each method is first measured. In method 1 , this includes the time to acquire road and facility data for the designated range, generate strokes, generate the fat stroke, and complete generalization. In method 2, this includes the time to acquire the necessary fat-stroke data from the precomputed fat-stroke DB and complete generalization.

\subsubsection{Results and Discussion}

Figure 13 compares the generalization execution times of Methods 1 and 2. The fat-stroke generation time increases with the size of the map owing to increase in the number of links and facilities. Therefore, in Method 1, the execution time increases rapidly with the size of the map. In the proposed method with the fat-stroke DB, fast generalization of the map is possible by referring to the fat stroke. The results suggest that in the proposed method, execution can be completed within $1 \mathrm{~s}$ regardless of the size of the map; thus, a viable response speed can be achieved for web map service.

The time for building the fat-stroke database was also measured separately. The time taken to generate strokes from road data is shown in Fig. 14 (a), and the breakdown of the time taken to link strokes with facilities and generate the fat stroke is shown in Fig. 14 (b). These can be precomputed and are not directly related to the system response time.

In summary, it takes a considerable amount of computation time to create the database in advance, but the response speed of the proposed method is sufficiently fast to realize the web map service. In Fig. 13, when the map size is within $1300 \times 1300 \mathrm{px}$, processing is completed within $1 \mathrm{~s}$. The screen resolution of a typical PC or smartphone is about $1280 \times 800$ or $1920 \times 1080 \mathrm{px}$, which means that processing will be completed within $2 \mathrm{~s}$ at the latest. Since processing of the road generalization is performed only once for each facility category search, we believe that the obtained response speed of 1 to $2 \mathrm{~s}$ is sufficiently fast. 


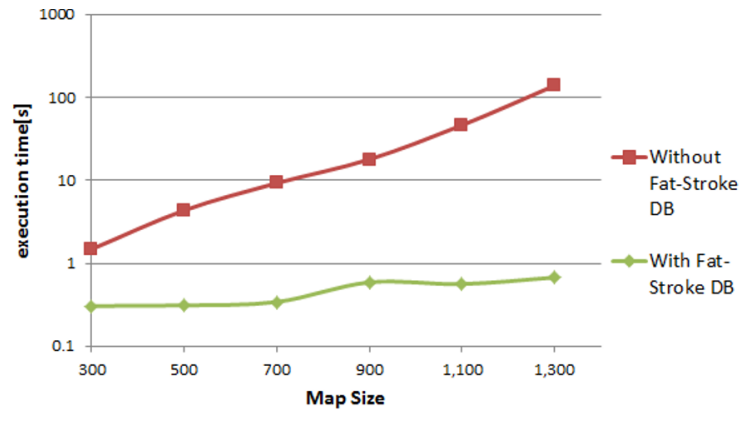

Fig. 13 Road generalization execution time

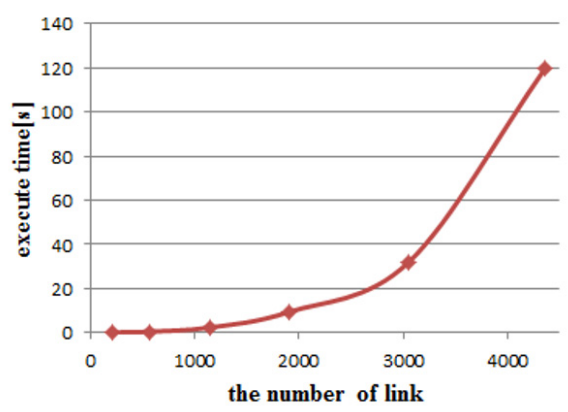

(a) Stroke generation time

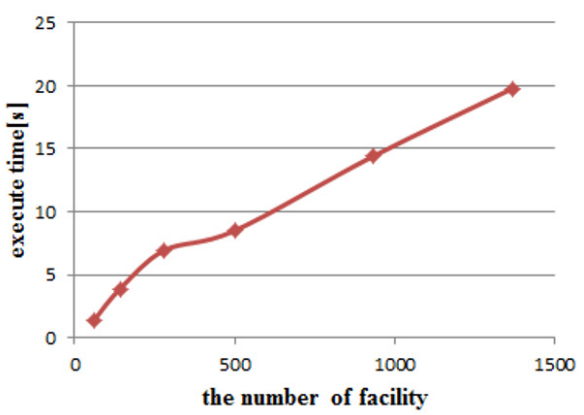

(b) Fat-stroke generation time

Fig. 14 Fat-stroke DB construction time

\subsection{Connectivity Evaluation}

As regards connectivity evaluation, Zhang used the connection between strokes as a criterion for evaluating road generalization [3]. This is because, as with real-world roads, the generalized roads must be connected to each other. In this study, because facility data were added to the stroke network, both the connectivity between strokes and the connectivity between strokes and facilities were used in the evaluation. Using these evaluation criteria, we compared the road generalization results of the conventional method and the proposed fat-stroke-based method.

\subsubsection{Evaluation Method}

To investigate the efficacy of the proposed method, we compared and evaluated the results obtained using three different methods: M1, M2, and M3.

Method M1 Using stroke length and facility information (proposed method) $\left(w_{f}=0.2, w_{s}=0.8\right)$

Method M2 Using only the stroke length (conventional method) $\left(w_{f}=0, w_{s}=1\right)$

Method M3 Using only the facility information $\left(w_{f}=1\right.$, $\left.w_{s}=0\right)$

The target area was centered at (latitude, longitude) $=$ $(35.157,136.930)$, with a map size of $7.989 \mathrm{~km} \times 7.989 \mathrm{~km}$. Facility data for "Parking" were used. The other parameters used were $s_{\text {width }}=5$ and $A=1024 \times 1024$ px. The number of strokes within the target area was 4416.

Two indices, $C_{S}$ and $C_{F}$, were defined for evaluating the connectivity in the road generalization by using the stroke set $S$ from the road generalization and the facility set $F$ associated with the stroke, wherein the number of elements in set $X$ is represented by $|X|$.

$$
\begin{aligned}
C_{S} & =\frac{1}{|S| L(S)} \sum_{s \in S} L(R(s)) \\
C_{F} & =\frac{1}{|F| L(S)} \sum_{f \in F} L(R(f))
\end{aligned}
$$

Here, function $R(s)$ affords a set of strokes that are reachable by tracing the connection to other strokes from stroke $s$, and function $R(f)$ affords a set of strokes that are reachable by tracing the connection to other strokes from a stroke linked to facility $f$. In addition, function $L(X)$ yields the total length of all the strokes in stroke set $X$. The equations for these functions are given below, where $s_{l e n}$ denotes the length of stroke $s$.

$$
\begin{aligned}
& R(s)=\left\{s^{\prime} \mid s^{\prime} \in S, s^{\prime} \text { is reacheable from } s\right\} \\
& R(f)=\left\{s^{\prime} \mid s^{\prime} \in S, s^{\prime} \text { is reacheable from } f\right\} \\
& L(X)=\sum_{s \in X} s_{\text {len }}
\end{aligned}
$$

Parameter $P_{\text {rate }}$, i.e., the proportion of the sum of the route lengths of the road generalization link set $P^{\prime}$, relative to the sum of the route lengths of the road link set $P$ actually existing within the map range, is given by the following equation, where $p_{\text {len }}$ denotes the length of road link $p$. All the roads are drawn when $p_{\text {rate }}=1$.

$$
P_{\text {rate }}=\frac{\sum_{p^{\prime} \in P^{\prime}} p_{\text {len }}^{\prime}}{\sum_{p \in P} p_{\text {len }}}
$$

\subsubsection{Results and discussion}

Figure 15 (a) shows the results of the $C_{S}$ computation while Fig. 15 (b) shows the results for $C_{F}$. The horizontal axis represents $P_{\text {rate }}$, the ratio of the total length of the roads in the generalization relative to that of the actual road network. As shown in Fig. 15 (a), the values of $C_{S}$ tend to be higher for M1 and M2 than for M3. These results suggest that in road generalization, M1 and M2 exhibit better connectivity than 
M3. Although the length of the stroke is used in M1 and M2 for calculating the importance of the stroke, in general, long strokes tend to intersect with each other, thereby improving the connectivity. In contrast, method M3 has a lower $C_{S}$ than those of the other methods. A long stroke is not necessarily chosen in M3, because the importance of the stroke is determined from the number of facilities only.

From Fig. 15 (b), we note that $C_{F}$ is greater for M1 and M3 than in M2. The results suggest that although M1 and M3 have a smaller number of strokes, more facilities are

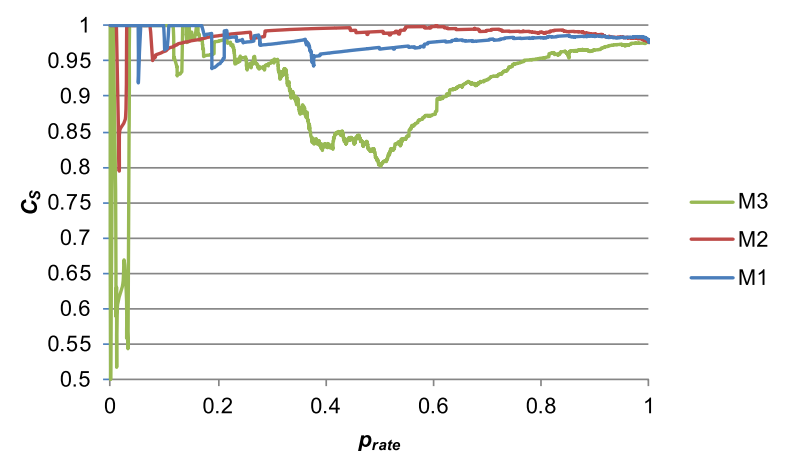

(a) Connectivity between strokes $\left(C_{S}\right)$

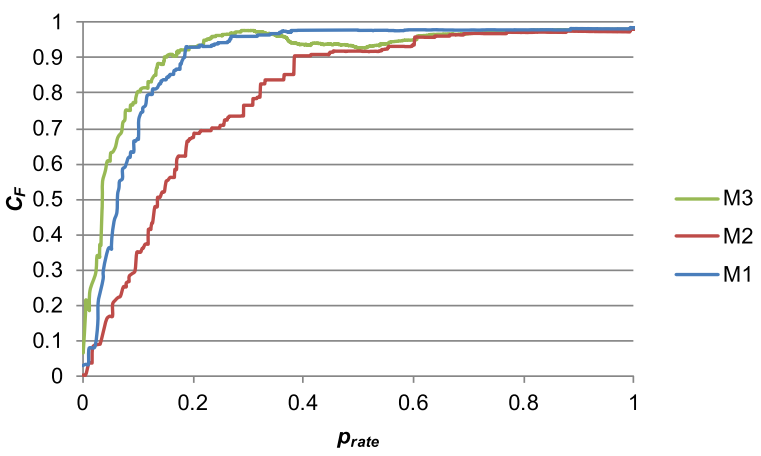

(b) Connectivity between strokes and facilities $\left(C_{F}\right)$

Fig. 15 Connectivity evaluation results reachable in the generalized road network. In contrast, the number of reachable facilities is reduced with $\mathrm{M} 2$ relative to the other methods because facility data are not considered in road selection.

Figure 16 shows the actual maps derived from road generalization using methods M1, M2, and M3. The red dots denote the facilities searched, and the black lines denote the strokes. Upon comparing methods M1 and M3, we observe that in M1, more long strokes are selected, and the strokes tend to be more interconnected. This supports the results of the evaluation index $C_{S}$. Upon comparing methods M1 and M2, we observe that with M1, more facilities are linked to strokes. This supports the results of evaluation index $C_{F}$.

Therefore, the results corresponding to indices $C_{S}$ and $C_{F}$ suggest that the proposed method M1can generate road generalization with high connectivity. In summary, considering the results presented in Sect.6.1.2, we can conclude that the proposed method realizes high-quality road generalization according to the facility search results while affording a high response speed as a web map service.

\section{Conclusion}

This study implemented an on-demand road generalization method that adapts to both the facility search results and the stroke length. Moreover, the proposed system can achieve a sufficiently fast response speed for web map services. In particular, we proposed a fat-stroke model that links facility information with strokes, a road generalization method based on the model, and a method to improve the response time. In addition, a prototype based on the proposed system was developed. The system evaluation results were based on three indicators, namely, response time of the road generalization system, connectivity between strokes, and connectivity between stroke and facilities. Our experimental results suggest that proposed method affords an improved response time by a factor of 100 or more. Further, when evaluating the connectivity between strokes and the connectivity be-

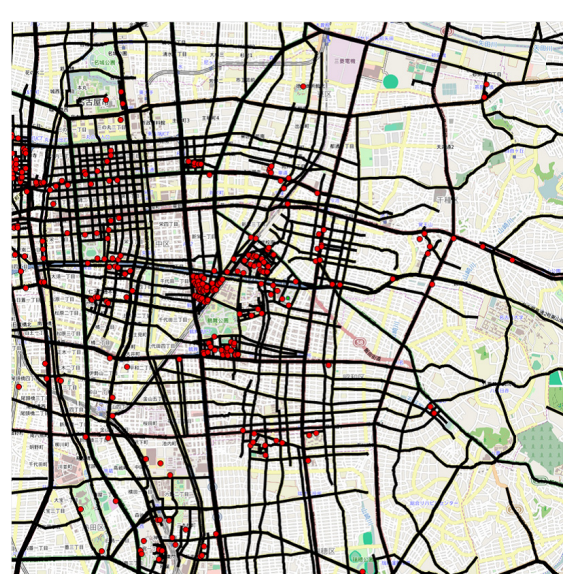

(a) Method M1

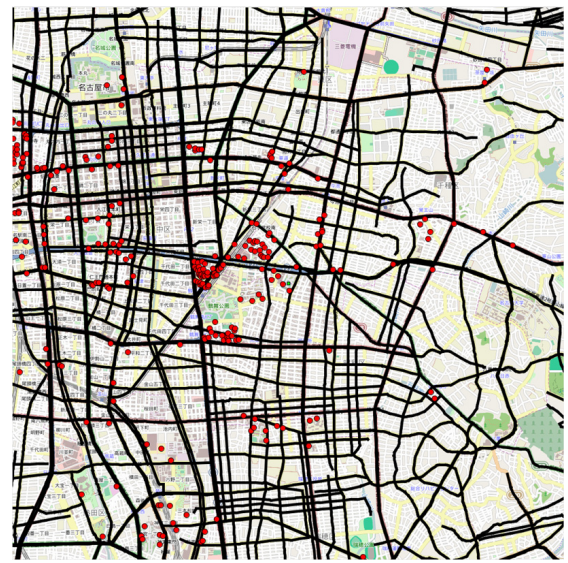

(b) Method M2

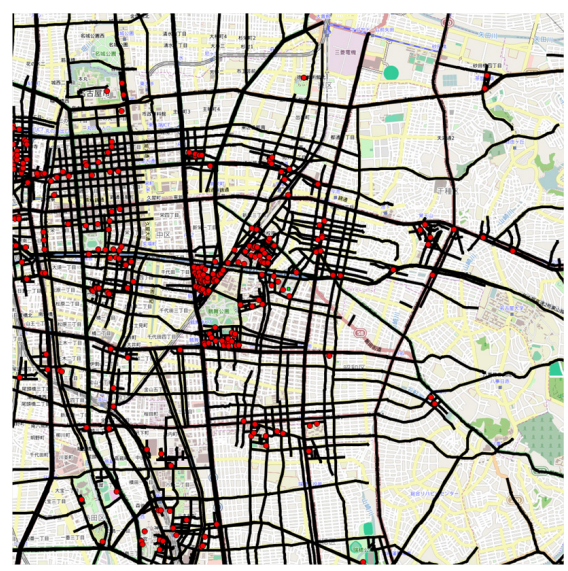

(c) Method M3

Fig. 16 Generalization results for $p_{\text {rate }}=0.3$ 
tween strokes and facilities, the method using the fat-stroke model was found to excel in both areas over conventional methods.

This study focused only on the implementation of road generalization; topics related to the drawing of strokes (color and thickness of lines, simplification) or the adjustment of stroke position, etc., as required in cognitive maps [20]-[22] and guide maps, are beyond the scope of this study. The mechanisms for taking these additional topics into consideration will be studied in the future. Moreover, although a system specific to Japan was developed for the experiments, the authors intend to extend the target area to cover other parts of the world and make the system freely available in the future.

\section{Acknowledgments}

This work was supported by JSPS KAKENHI Grant Numbers JP26330136, JP25700009 and SCOPE.

\section{References}

[1] R. Thomson and R. Brooks, "Efficient generalisation and abstraction of network data using perceptual grouping," Proc. 5th International Conference on GeoComputation, pp.23-25, 2000.

[2] R. Thomson and D. Richardson, "'Good continuation' principle of perceptual organization applied to the generalization of road networks, Proc. 19th International Cartographic Conference, pp.12151223, 1999.

[3] Q. Zhang, "Road network generalization based on connection analysis," Proc. 11th Interantional Symposium on Spatial Data Handling, pp.343-353, 2005.

[4] M. Murase, D. Yamamoto, and N. Takahashi, "On-demand generalization of guide maps with road networks and category-based web search results," Proc. W2GIS 2015, pp.53-70, April 2015.

[5] K. Fujii and K. Sugiyama, "Route guide map generation system for mobile communication," Information Processing Society of Japan Journal, vol.41, no.9, 2394-2403, 2000.

[6] T. Nagata and Y. Maeda, "Agent-based geographic information selection in the deformed map," Proc. Human Interface Symposium 2006, pp.479-484, 2006.

[7] K. Maruyama, M. Tanizaki, and S. Shimada, "Mathematical transformation model of road network for deformed map generation and its system evaluation," Proc. Institute of Electronics Information and Communication Engineers Technical Report, ITS2002-192, pp.7784, 2003.

[8] J.T. Bjrke, "Generalization of road networks for mobile map services: an information theoretic approach," Proc. 21st International Cartographic Conference, pp.127-135, 2003.

[9] Y. Hu, J. Chen, Z. Li, and R. Zhao, "Selection of streets based on mesh density for digital map generalization," Proc. Fourth International Conference on Image and Graphics (ICIG 2007), pp.903-908, 2007.

[10] B. Jiang and C. Claramunt, "A structual approach to the model generalization of an urban street network," GeoInformatica, vol.8, no.2, pp.157-171, June 2004.

[11] S. Ravada, X. Chen, Z. Liu, and X. Zhang, "Sig spatial cup report: constrained map generalization," Proc. 22nd ACM SIGSPATIAL International Conference on Advances in Geographic Information Systems, pp.605-608, 2014.

[12] D. Yamamoto, S. Ozeki, and N. Takahashi, "Focus+Glue+Context: an improved fisheye approach for web map services," Proc. ACM SIGSPATIAL GIS 2009, pp.101-110, 2009.
[13] N. Ninomiya, N. Togawa, M. Yanagisawa, and T. Ohtsuki, "A deformed map generation algorithm for small displays based on cognitive science and its stochastic evaluations," IEICE Trans. Fundamentals (Japanese Edition), vol.J91-A, no.9, pp.869-882, 2008.

[14] K. Kono, T. Nitta, K. Ishikawa, M. Yanagisawa, and N. Togawa, "Comprehensive deformed map generation for wristwatch-type wearable devices based on landmark-based partitioning," Proc. 2016 IEEE 5th Global Conference on Consum. Electron., 2016.

[15] J. Matsuo, D. Kitayama, and K. Sumiya, "An extracting method of dependencies of geographical and image features for retrieving artificial maps and its applications," IPSJ Transactions on Databases, vol.6, no.3, pp.90-104, 2013.

[16] Z. Wei, J. He, L. Wang, Y. Wang, and Q. Guo, "A collaborative displacement approach for spatial conflicts in urban building map generalization," IEEE Access, vol.6, pp.26918-26929, May 2018.

[17] B. Zhao, D. Peng, S. Hu, and S. Liu, "A practical technique to solve settlements overlapping roads problem in map generalization," Proc. 2015 23rd International Conference on Geoinformatics, 2015.

[18] J. Lee, H. Jang, J. Yang, and K. Yu, "Machine learning classification of buildings for map generalization," ISPRS Int. J. Geo-Information, vol.6, no.10, 309, 2017.

[19] D. Yamamoto, H. Itoh, and N. Takahashi, "One click focusing: an SQL-based fast loop road extraction method for mobile map service," Proc. 4th International Conference on Advanced Geographic Information Systems, Applications, and Services, pp.7-16, 2012.

[20] P. Gould and R. White, "Mental maps," Penguin Books Ltd, Harmondsworth, Middlesex, England, 1974.

[21] K. Lynch, "The image of the city," MIT Press, Cambridge, 1960.

[22] E.C. Tolman, "Cognitive maps in rats and men," The Psychological Review, vol.55, no.4, pp.189-208, 1948.

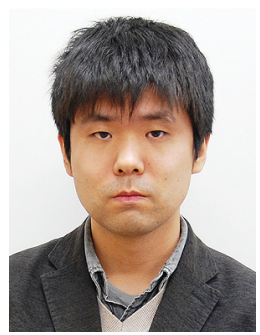

Daisuke Yamamoto is an associate professor in the Information Technology Center at Nagoya Institute of Technology, Japan. He received a Ph.D. in Information Science from Nagoya University. His research interests include Web services, Web interaction, content technologies, e-learning, geographical information systems, and multimedia systems. Dr. Yamamoto is a member of the IEEE, the Information Processing Society of Japan, the virtual reality society of Japan, and the Database Soci-

ety in Japan.

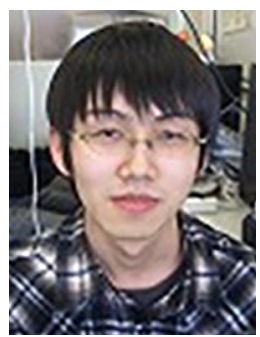

Masaki Murase works for Tokai Rika Co., LTD. He received the B.S. and M.S. degrees in Electrical Engineering from Nagoya Institute of Technology. 


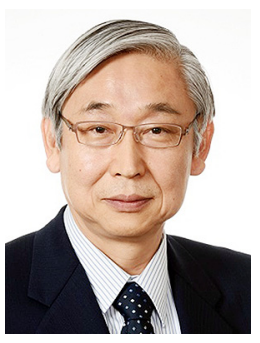

Naohisa Takahashi is a professor emeritus and a project professor, Nagoya Institute of Technology, since April 2017. He worked for the Department of Computer Science at NIT as a professor from April 2001 to March 2017. Prior to coming to NIT, he was engaged in research and development at NTT Laboratories for 25 years. He received B.E. and M.E. degrees in electrical engineering from the University of Electro-Communications, Tokyo, Japan, in 1974 and 1976, respectively. He received a doctorate in computer science in 1987 from Tokyo Institute of Technology. His recent research interests are network computing, ubiquitous computing, geographical information systems and e-learning systems. Dr. Takahashi is a member of the IEEE, the Association for Computing Machinery, the Information Processing Society of Japan, and the Database Society in Japan. 